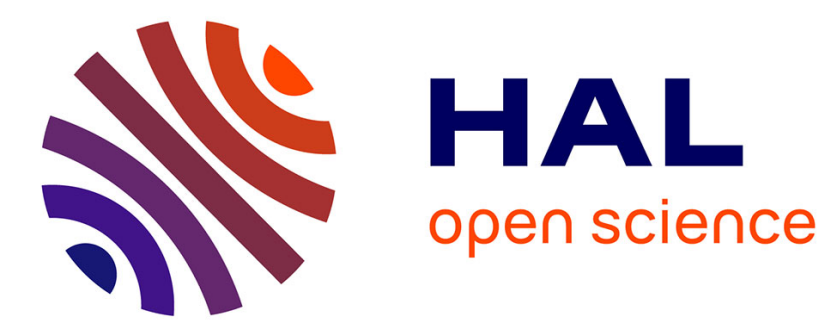

\title{
Spontaneous seizure remission following status epilepticus in drug-resistant epilepsy due to focal cortical dysplasia
}

Nadine Girard, Eric Guedj, Patrick Chauvel, Fabrice Bartolomei, Aileen Mcgonigal

\section{To cite this version:}

Nadine Girard, Eric Guedj, Patrick Chauvel, Fabrice Bartolomei, Aileen Mcgonigal. Spontaneous seizure remission following status epilepticus in drug-resistant epilepsy due to focal cortical dysplasia. Epilepsy Research, 2017, 137, pp.73-77. 10.1016/j.eplepsyres.2017.09.013 . hal-02479808

\section{HAL Id: hal-02479808 https://hal.science/hal-02479808}

Submitted on 6 Apr 2020

HAL is a multi-disciplinary open access archive for the deposit and dissemination of scientific research documents, whether they are published or not. The documents may come from teaching and research institutions in France or abroad, or from public or private research centers.
L'archive ouverte pluridisciplinaire HAL, est destinée au dépôt et à la diffusion de documents scientifiques de niveau recherche, publiés ou non, émanant des établissements d'enseignement et de recherche français ou étrangers, des laboratoires publics ou privés. 


\title{
Spontaneous seizure remission following status epilepticus in drug- resistant epilepsy due to focal cortical dysplasia
}

\author{
*Nadine Girard ${ }^{1}$, *Eric Guedj 2, 3, 4, Patrick Chauvel ${ }^{5,6}$, Fabrice Bartolomei ${ }^{6,7}$, **Aileen \\ McGonigal ${ }^{6,7}$
}

${ }^{1}$ Department of Neuroradiology, Aix-Marseille University, AP-HM, Marseille, France

${ }^{2}$ Department of Nuclear Medicine, Assistance Publique-Hôpitaux de Marseille, Aix-Marseille University, Timone University Hospital, France

${ }^{3}$ CERIMED, Aix-Marseille University, Marseille, France

${ }^{4}$ Institut de Neurosciences de la Timone, UMR 7289, CNRS Aix-Marseille Université, Marseille, France.

${ }^{5}$ Neurological Institute Epilepsy Center, Cleveland Clinic, Cleveland OH 44195.

${ }^{6}$ Aix Marseille University, Institut de Neurosciences des Systèmes, UMR 1106, Marseille, France

${ }^{7}$ Department of Clinical Neurophysiology, Hôpital de la Timone, Assistance Publique-Hôpitaux de Marseille, Marseille, France

*Authors equally contributed

**Corresponding author

\section{Summary}

We describe a patient with chronic pharmacoresistant epilepsy related to right parietal focal cortical dysplasia (FCD), who became seizure-free following an episode of convulsive status epilepticus (SE). Magnetic resonance imaging (MRI) and fludeoxyglucose positron emission tomography (FDG-PET) were performed before and after SE. Longitudinal MRI scans showed a stable appearance of the FCD with no new signal change. However, diffusion tensor imaging showed altered white matter fiber tract orientation in posterior cortices, especially in proximity to the lesion, at 3 years post-SE. FDG-PET showed more widespread hypometabolism 3 years after SE. The unusual occurrence of spontaneous seizure remission following SE in the context of FCD-related epilepsy, in association with neuroimaging evolution, suggests possible cerebral reorganization triggered by SE as a mechanism in this case.

Key words

Focal cortical dysplasia; status epilepticus; FDG-PET; diffusion tensor imaging 
Focal cortical dysplasias (FCD) are typically associated with epilepsy that often begins in childhood, the majority of cases proving to be pharmacoresistant ${ }^{1}$. Because of poor response to antiepileptic drug (AED) treatment, surgery is often required, and spontaneous remission of epilepsy without surgery in these cases is very rare $^{2}$.

We report the case of a patient with chronic drug-resistant epilepsy related to focal cortical dysplasia, in whom an episode of convulsive status epilepticus (SE) was followed by complete and sustained remission of seizures, with longitudinal modifications in neuroimaging.

\section{Clinical case}

A 27-year-old right handed woman first presented seizures as an infant aged 8 months with eyelid clonus. Seizure expression evolved to altered contact and hypotonic falls by age 18 months. A period of seizure freedom was achieved with vigabatrin, taken until age 6 years. The patient remained seizure free off anti-epileptic drugs (AED) till aged 9 years, at which time seizures re-appeared, mainly nocturnal, characterised by unpleasant cephalic sensation, pressure in throat then loss of consciousness with hyperkinetic movements of all 4 limbs and ocular clonus, often progressing to secondary generalised tonic-clonic seizures (GTCS). Cerebral magnetic resonance imaging (MRI) performed at age 14 years in 2004 demonstrated right parietal focal cortical dysplasia (FCD), likely Type II (Fig 1A) and fludeoxyglucose positron emission tomography (FDG-PET) showed hypometabolism in the same region (Fig 2A). Because of resistance to AED polytherapy, presurgical evaluation was undertaken, including stereoelectroencephalography (SEEG) in 2010, which showed interictal discharges in relation to the FCD. However, despite prolonged SEEG recording during 3 weeks, only one spontaneous seizure was recorded, which showed some non-habitual semiological features and no clear electrophysiological change preceding clinical onset. The result of SEEG was therefore considered inconclusive and insufficient to permit surgical planning. Although a second SEEG exploration was subsequently proposed the patient declined this, and treatment was thereafter based on medical therapy alone. Transient improvement in seizure frequency and severity was seen during the 2 
months following SEEG, but her epilepsy remained active with seizure frequency of several nocturnal seizures per month, often secondary GTCS. She also developed fairly disabling migraines around this time. While baseline cognitive function was normal, her epilepsy severity required that stopping her university course. Multiple unsuccessful combinations of AED were tried, the most efficacious being topiramate and vigabatrin. Topiramate had a beneficial effect on migraine frequency. No period of seizure freedom of more than 14 days occurred between ages 9 and 24 years.

In 2013 at the age of 24 years, during a febrile illness, the patient presented a cluster of nocturnal GTCS evolving to generalized convulsive status epilepticus. This required emergency intensive care management with intubation and intravenous benzodiazepines and barbiturates, with resolution of seizures and extubation 12 hours later. Cerebral computerized tomography and cerebrospinal fluid examination were normal; no infectious cause was identified. Electroencephalography 48 hours after termination of SE showed persistent bilateral slow and spike activity, predominant on the right. Following this episode of SE, the patient recovered rather gradually, complaining of ataxia and vertigo during a period of several weeks. The EEG in the month following SE remained altered with regards to baseline EEG, showing bilateral diffuse slow wave activity. Structural MRI was unchanged (Fig 1C). Clinical state and EEG abnormalities at this time were considered probably related to valproate therapy, which had been added after the episode of SE, with elevated plasma levels initially. At this point she was taking sodium valproate, topiramate, clobazam and vigabatrin. With AED dose reduction she gradually returned to her normal state with no neurological or cognitive sequellae and EEG normalized, at around 6 weeks after the episode of SE. Valproate was subsequently discontinued due to poor tolerance. In the 6 months following SE, the patient herself reduced and then stopped both vigabatrin and clobazam. In 2014, 1 year after SE, she also (without medical advice) reduced topiramate to $25 \mathrm{mg}$ per day, which remains her current regimen as monotherapy. No seizures have occurred since SE at 40 months' follow-up and the patient now works as a research assistant in a university laboratory. She reports noticeable improvement in quality of life, mood and cognitive function since post-SE seizure cessation and AED reduction. Cerebral MRI performed 36 months after SE (Fig1D) showed no structural change in relation to SE, and the appearance of the FCD remained similar to earlier imaging (fig 1A-C). However, diffusion tensor imaging (DTI) colored fractional 
anisotropy map showed marked change in posterior cortex white matter tract orientation at 36 months post-SE compared to 1 month post-SE (fig 1D), suggesting cerebral reorganization in relation to the episode of SE. No DTI images were obtained prior to SE. Cerebral FDG-PET performed 36 months after SE showed alteration compared 6 years earlier (fig 2), with increased extent and severity of right posterior regional hypometabolism.

\section{Discussion}

This observation is a rare illustration of seizure remission occurring in a patient with chronic drugresistant epilepsy due to radiologically confirmed FCD, in whom the temporal relation between episode of SE and seizure cessation suggests a likely causal role of SE.

Complete seizure remission lasting $>12$ months in chronic pharmacoresistant epilepsy, including cases of FCD, may be seen in $4-10 \%$ of patients without surgery ${ }^{3}$ but usually occurs in a context of AED change $^{2}$. Radiologic findings in our patient indicated FCD (likely type II) as epilepsy etiology, with consistent regional PET changes ${ }^{4}$, and SEEG recording confirmed interictal abnormalities and subclinical discharges typical of this diagnosis ${ }^{5}$. Presence of FCD as etiology does not per se seem to infer greater or lesser chance of such spontaneous remission occurring, but likelihood of attaining subsequent seizure freedom has been shown to be rather decreased if SE occurs in adults during their epilepsy history ${ }^{2}$. This presumably reflects more severe epilepsy cases, but may also indicate pathophysiological sequellae of SE with tendency to extension of epileptic networks, as has been shown in experimental models of brain circuitry ${ }^{6}$. Dysplastic cortex is intrinsically hyperexcitable, and cerebral networks associated with FCD are recognized to be highly epileptogenic and associated with electrophysiologic relationships that may be complex and unpredictable ${ }^{7}$. In patients with pharmacoresistant epilepsy related to FCD, surgical excision of the whole dysplastic network is typically required in order to achieve seizure freedom ${ }^{7}$. The present observation of spontaneous seizure cessation in a case of FCD following SE is thus highly unusual, and poses intriguing questions as to possible mechanisms involved. 
Following SE, MRI showed no change in the appearance of the dysplasia itself, nor new gray or white matter signal changes elsewhere, but changes in white matter tract orientation were demonstrated. A clear difference in white matter tract orientation in bilateral posterior cortices was seen in FA images between 1 month after SE and 3-years post-SE, suggesting an evolving process of cortical reorganization. While the lack of baseline FA data prior to SE limits somewhat the conclusions that can be drawn, the time course of these changes is highly suggestive of a causal role for SE. Diffusion tensor imaging (DTI) and fractional anisotropy (FA) have previously been used to demonstrate altered connectivity in subcortical tissue surrounding $\mathrm{FCD}^{8}$. Increased regional hypometabolism on FDG-PET compared to the pre-SE exam could also be in keeping with neuronal reorganization, although various factors such as cessation of seizure activity and AED reduction could equally contribute to the metabolic changes seen here.

The organisation of epilepsies related to FCD has been elucidated by SEEG studies that have led to recognition of particular electrophysiological signatures of interictal and ictal activity ${ }^{5,9}$. In terms of network organization, signal analysis shows that in Type II FDC, the dysplasia plays a main role as a hub within the network, directly contributing to seizure initiation and propagation of seizures ${ }^{10}$. This typically lesion-centric network organization helps explain the often excellent surgical outcome in Type II FCD, whether radiologically visible or not, in contrast to some other cortical developmental pathologies (such as heterotopia), in which the epileptogenic network is often more widespread and not necessarily centered on the lesion.

While little human imaging data on long-term outcome of SE exist, MRI studies following SE in rat models show structural changes preferentially in cortex, amygdala and hippocampus, and changes in cerebral blood volume that are strikingly regional, involving specific layers of parietal and cingulate cortex as well as hippocampus, thalamus and striatum ${ }^{11}$, suggesting different thresholds in different brain structures for expressing sequellae of SE. SE is well known to produce tissue damage that may lead to secondary epileptogenicity especially in hippocampus ${ }^{12}$, and vascular changes during SE are likely to contribute to this complex process ${ }^{13}$. On the other hand the initial damage induced by SE can also enhance neuroplasticity as a recovery process ${ }^{14}$. A role for vascular density and blood brain barrier integrity in relation to epileptogenicity has been suggested based on observations from animal 
models of epileptogenesis ${ }^{15}$, not only in terms of pathological effect but also in promoting beneficial outcome following cerebral injury ${ }^{14}$.

While clearly no conclusion can be drawn from this single case report, we might speculate that neuronal reorganization related to SE in the present case produced modulation of the dysplastic network that then led to seizure cessation, possibly via a process of cortical deafferentation, and perhaps augmented by higher sensitivity of parietal lobes to the effects of SE. The degree to which the imaging changes reported here reflect such a process is of course uncertain. However, this isolated and unusual case highlights the need for ongoing work into pathophysiological mechanisms involved in SE and epileptogenesis, including the impact of vascular and metabolic changes on epileptic networks. 


\section{Legends to Figures}

\section{Figure 1}

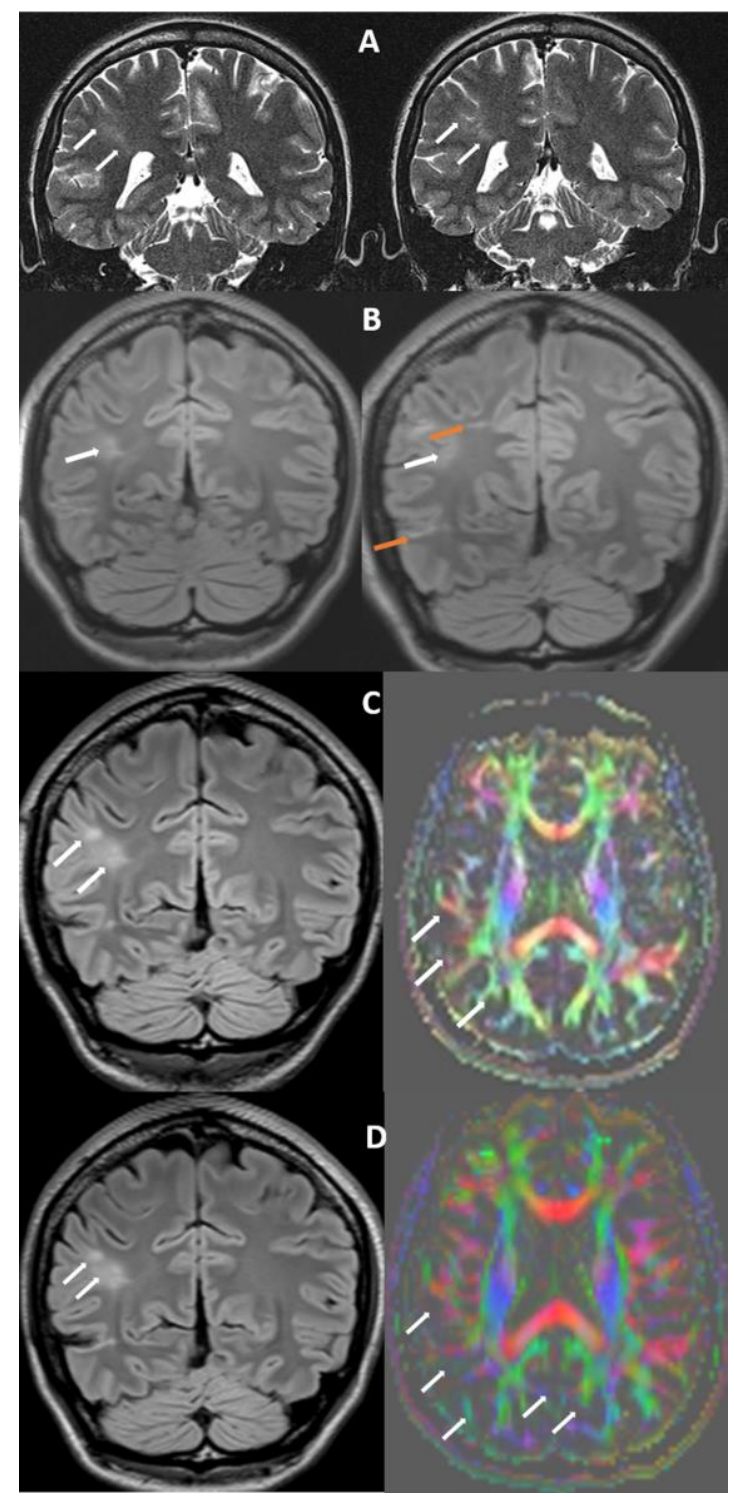

Fig 1 A. One year prior to SEEG: coronal T2 images showing focal cortical dysplasia characterized by slightly increased signal that extends from the cortex to the lateral ventricle (white arrows).

Fig 1B. Eighteen months after SEEG (before SE): coronal FLAIR images. Focal cortical dysplasia showing bright signal, similar to prior MRI (white arrow). Also note linear increased signal above the FCD, related to SEEG electrode (orange arrows)

Figure 1C. One month after SE. Left image: coronal FLAIR image showing similar appearance of FCD. Right image: axial color FA image showing posterior asymmetry, with different orientation of white matter tracts underlying the cortical abnormality, compared to contralateral region.

Fig 1D. Three years after SE. Left image: coronal FLAIR image showing similar appearance of FCD. Right image: axial color FA image showing modification of the orientation of white matter tracts in right posterior cortex underlying the FCD but also contralateral mesial posterior cortex, compared to 
the previous MRI shown in Fig 1C. Please note that colour intensity in figure 1D compared to the paler figure $1 \mathrm{C}$ is different simply because of technical changes in MRI acquisition in our center in the time period between these 2 scans. The important feature is the change in position of colored fibers, representing altered orientation of white matter tracts. 


\section{Figure 2}

Axial brain 18-FDG PET performed before (Fig 2A) and 38 months after (Fig2B) the status epilepticus; 3-slices coregistration and fusion with 3F Flair MRI (Fig 2C). Focal inferior parietal PET hypometabolism was initially found, with more posterior diffuse abnormalities after the status epilepticus.
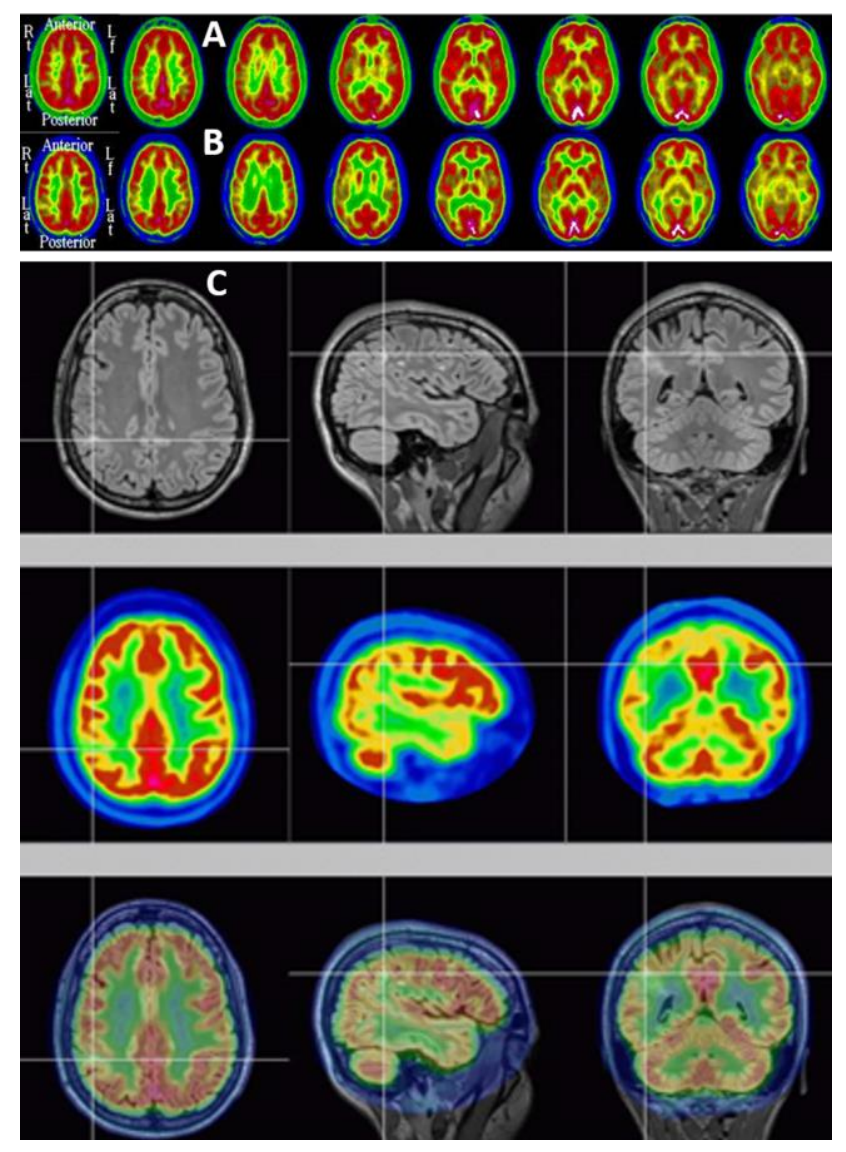


\section{Acknowledgements}

This paper has been carried out within the Federation Hospitalo-Universitaire (FHU) EPINEXT thanks to the support of the A*MIDEX project (ANR-11-IDEX-0001-02) funded by the "Investissements d'Avenir" French Government program managed by the French National Research Agency (ANR).

\section{Conflict of interest}

None of the authors has any conflict of interest to disclose concerning the present publication.

We confirm that we have read the Journal's position on issues involved in ethical publication and affirm that this report is consistent with those guidelines.

1. Semah F, Picot M-C, Adam C, et al. Is the underlying cause of epilepsy a major prognostic factor for recurrence? Neurology. 1998;51:1256-62.

2. Callaghan BC, Anand K, Hesdorffer D, et al. Likelihood of seizure remission in an adult population with refractory epilepsy. Annals of neurology. 2007;62:382-9.

3. Choi $H$, Heiman $G$, Pandis $D$, et al. Seizure remission and relapse in adults with intractable epilepsy: a cohort study. Epilepsia. 2008;49:1440-5.

4. Salamon N, Kung J, Shaw S, et al. FDG-PET/MRI coregistration improves detection of cortical dysplasia in patients with epilepsy. Neurology. 2008;71:1594-601.

5. Chassoux F, Devaux B, Landré E, et al. Stereoelectroencephalography in focal cortical dysplasia A 3D approach to delineating the dysplastic cortex. Brain. 2000;123:1733-51.

6. Morimoto K, Fahnestock M, Racine RJ. Kindling and status epilepticus models of epilepsy: rewiring the brain. Progress in neurobiology. 2004;73:1-60.

7. Duchowny M. Clinical, functional, and neurophysiologic assessment of dysplastic cortical networks: implications for cortical functioning and surgical management. Epilepsia. 2009;50:19-27.

8. Lee S-K, Kim DI, Mori S, et al. Diffusion tensor MRI visualizes decreased subcortical fiber connectivity in focal cortical dysplasia. Neuroimage. 2004;22:1826-9.

9. Lagarde S, Bonini F, McGonigal A, et al. Seizure-onset patterns in focal cortical dysplasia and neurodevelopmental tumors: Relationship with surgical prognosis and neuropathologic subtypes. Epilepsia. 2016;57:1426-35.

10. Varotto G, Tassi L, Franceschetti S, et al. Epileptogenic networks of type II focal cortical dysplasia: a stereo-EEG study. Neuroimage. 2012;61:591-8.

11. Fabene $P$, Marzola $P$, Sbarbati $A$, et al. Magnetic resonance imaging of changes elicited by status epilepticus in the rat brain: diffusion-weighted and T2-weighted images, regional blood volume maps, and direct correlation with tissue and cell damage.

Neuroimage. 2003;18:375-89. 
12. Wasterlain CG, Fujikawa DG, Penix L, et al. Pathophysiological mechanisms of brain damage from status epilepticus. Epilepsia. 1993;34.

13. Choy $M$, Wells J, Thomas $D$, et al. Cerebral blood flow changes during pilocarpineinduced status epilepticus activity in the rat hippocampus. Experimental neurology. 2010;225:196-201.

14. Gröhn O, Sierra A, Immonen R, et al. Multimodal MRI assessment of damage and plasticity caused by status epilepticus in the rat brain. Epilepsia. 2011;52:57-60.

15. Ndode-Ekane X, Hayward N, Gröhn O, et al. Vascular changes in epilepsy: functional consequences and association with network plasticity in pilocarpine-induced experimental epilepsy. Neuroscience. 2010;166:312-32. 